\title{
Interactive comment on "Sea ice export through the Fram Strait derived from a combined model and satellite data set" by Chao Min et al.
}

\section{Anonymous Referee \#1}

Received and published: 21 August 2019

\section{Overall:}

In this manuscript the authors use a recently published CMST data product on Arctic sea ice thickness and drift to derive and analyze a variability of Fram Strait sea ice export over the period of 2010-2016.

The manuscript is clearly written and results are also presented well. The provided estimates of melt season volume fluxes is definitely a strong side of the entire story.

My only moderate (to major) concern is related with the use of the $82 \mathrm{~N}$ gate alone to calculate the sea ice export from the Arctic. Though a resulted lower uncertainty as a main motivation (presented in Fig. 12) is apparent, given their new data product, it should be relatively straightforward and highly beneficial for this work to run the same 
computations for the $79 \mathrm{~N}$ transect. This will allow to consider these new results in the context of earlier/similar studies on the topic, e.g. Kwok et al., 2004, Spreen et al., 2009. The authors already refer to Kwok et al., 2004 when discussing and comparing the seasonal/annual volume fluxes and their changes over two decades; a more direct comparison of the two products with respect to the volume fluxes would be possible if the fluxes at $79 \mathrm{~N}$ were presented as well. Therefore, in addition to their flux estimates for the northern gates, the authors are encouraged to extend their calculations to a southerly transect at $79 \mathrm{~N}$ which accommodates the FS ULSs.

In general, the manuscript deserves to be published after these relatively moderate modifications to the content. Some minor corrections are also suggested in the list of minor comments below.

\section{Minor comments:}

Line 20: please round off to significant figures throughout the text, e.g. $244+-43$ can well be rounded to $240+-40$ etc

Line 51: "In terms of sea ice volume flux, Ricker et al. (2018) and Bi et al. (2018)..." one can site Zamani et al., 2019 (https://doi.org/10.1007/s00382-019-04699-z) too.

Lines $75-88$. Pease indicate in 2.1. that CMST data in addition to ice thickness and concentration also comprises the modelled/assimilated ice drift velocities.

Line 106: Please cite HEM data properly, refer to Renner at al., $2014(10.1002 / 2014$ GL060369) and a respective data citation found in (https://data.npolar.no/dataset/1ed8c57e-8041-42fd-95bb-cfe4e181e9b8)

Line 133: please state explicitly "meridional velocity" and "zonal velocity" or "meridional and zonal components of the sea ice drift vector"

Line 181: "thinning trend from west to east", one can mention this is in line with other studies on the topic (e.g. Kwok et al., Hansen et al., Krumpen et al., etc). 
Line 187: "within the Arctic basin"?

Line 197: ". . slightly smaller than that of CS2". This is certainly not the case; the discrepancies between the data sets as presented in Figure 5 can not be referred to as "slightly smaller".

Line 209: “. . .consistent with previous studies, such as. .." pointed already in Kwok, et al.

Sections 3.3 and 3.4. As I pointed earlier, having sea ice volume fluxes estimated at $79 \mathrm{~N}$ too, would have made a comparison with other studies much more straightforward. No need to add more figures, the additional data can well be accommodated in the existing ones.

Interactive comment on The Cryosphere Discuss., https://doi.org/10.5194/tc-2019-157, 2019. 